This item was submitted to Loughborough's Research Repository by the author.

Items in Figshare are protected by copyright, with all rights reserved, unless otherwise indicated.

\title{
On step approximations for water-wave problems
}

PLEASE CITE THE PUBLISHED VERSION

PUBLISHER

(c) Cambridge University Press

VERSION

VoR (Version of Record)

LICENCE

CC BY-NC-ND 4.0

REPOSITORY RECORD

Evans, D.V., and C.M. Linton. 2019. "On Step Approximations for Water-wave Problems". figshare. https://hdl.handle.net/2134/4263. 
This item was submitted to Loughborough's Institutional Repository (https://dspace.lboro.ac.uk/) by the author and is made available under the following Creative Commons Licence conditions.

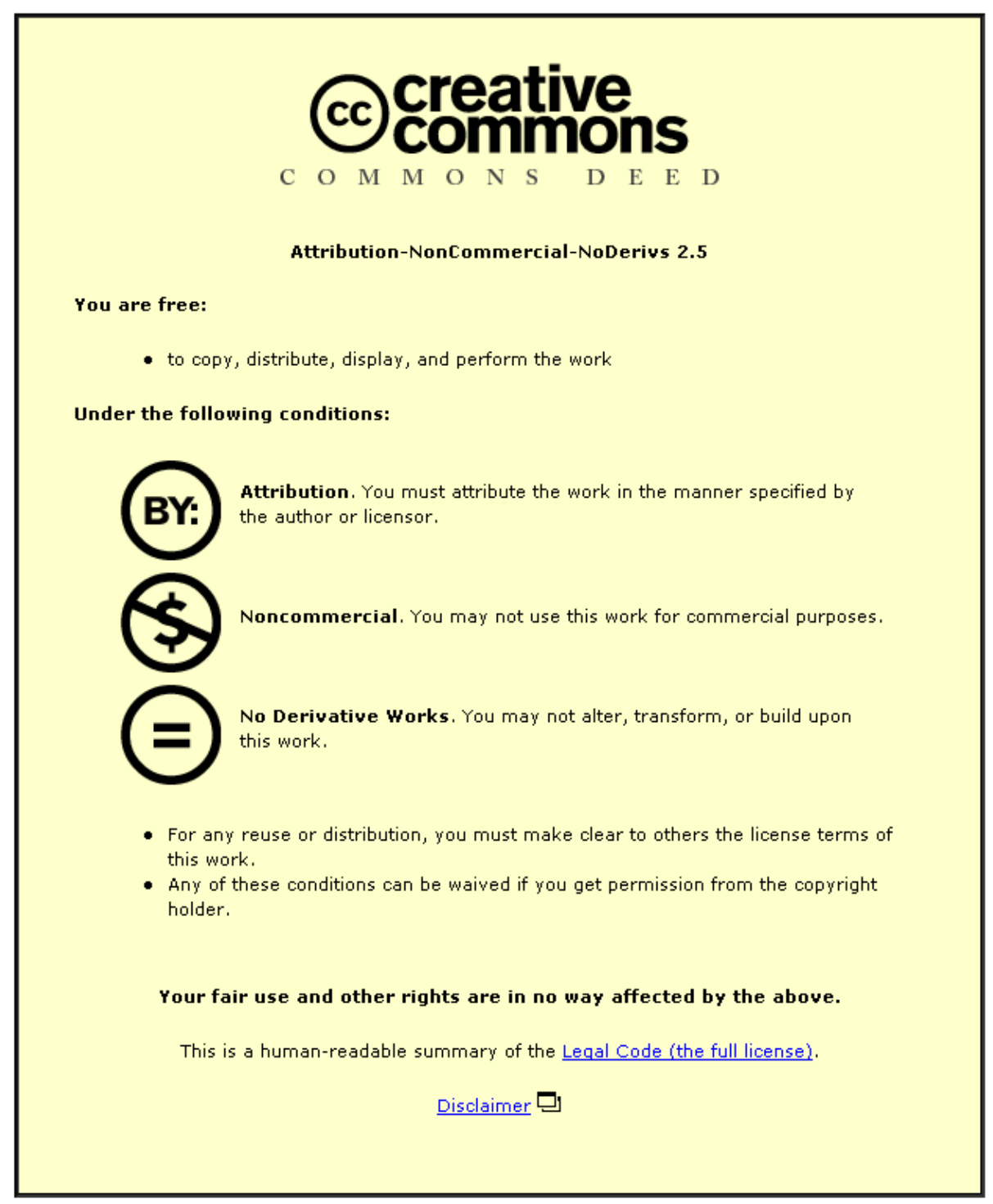

For the full text of this licence, please go to: http://creativecommons.org/licenses/by-nc-nd/2.5/ 


\title{
On step approximations for water-wave problems
}

\author{
By D. V. EVANS ${ }^{1}$ AND C.M. LINTON ${ }^{2}$ \\ ${ }^{1}$ School of Mathematics, University of Bristol, Bristol BS8 1TW, UK \\ ${ }^{2}$ Department of Mathematical Sciences, Loughborough University of Technology, Leicestershire, \\ LE11 3TU, UK
}

(Received 10 September 1993 and in revised form 14 March 1994)

The scattering of water waves by a varying bottom topography is considered using two-dimensional linear water-wave theory. A new approach is adopted in which the problem is first transformed into a uniform strip resulting in a variable free-surface boundary condition. This is then approximated by a finite number of sections on which the free-surface boundary condition is assumed to be constant. A transition matrix theory is developed which is used to relate the wave amplitudes at $\pm \infty$. The method is checked against examples for which the solution is known, or which can be computed by alternative means. Results show that the method provides a simple accurate technique for scattering problems of this type.

\section{Introduction}

The problem of determining the scattering of a long-crested gravity wave over a bottom of arbitrary shape has received considerable attention in the literature. For a survey up to 1960 see Wehausen \& Laitone (1960, p.525ff). Despite the simplification afforded by linearization of the free-surface condition, there appears to be only one explicit solution for a particular bottom shape. This solution, due to Roseau (1976), provides an explicit representation for the two-dimensional velocity potential of the field produced by a time-harmonic plane wave incident upon an underwater bottom topography having a smooth variation, in the direction of wave propagation only, from one constant depth to another.

For general bottom topographies numerical methods are available. For geometries where the depths at either side tend asymptotically to the same value, a solution can be obtained by utilizing the Green's function for a time-harmonic wave source in finite depth. Application of Green's theorem provides a Fredholm integral equation for the unknown velocity potential over the non-constant part of the bottom profile whilst a separate integral representation provides the potential at an arbitrary point in the fluid region in terms of the potential on this bottom profile. Despite the simplicity of this formulation the authors are not aware of any calculations using this method for a given bottom profile, although the method has been used in a corresponding acoustic problem to derive frequencies of trapped modes over an arbitrary obstacle in an acoustic waveguide (Linton \& Evans 1992).

Of more interest, and more difficulty, is the problem of the bottom profile joining regions of different constant depths, since this corresponds to waves propagating over, for instance, a continental shelf of a given shape. The numerical technique described above is no longer available because of the difference in depths. This would need to be modified by using a Green's function appropriate to this case and again deriving 
an integral equation over the variable part of the bottom profile. Such a function has been developed (Evans 1972) for precisely this purpose, but its complicated nature makes the numerical procedure unwieldy.

Recently boundary element methods have been used by several authors in connection with problems of wave propagation over arbitrary topographies. The complicated wave source discussed above is replaced by the simpler free-space Green's function, resulting in an integral equation for the unknown potential over the free surface and bottom. For example, Nachbin \& Papanicolaou (1992a) have used the method to consider the propagation of waves in shallow water over a random topography of arbitrary shape and slope. For the case of scattering by a bottom profile joining two regions of unequal depth, a hybrid method can be used in which the velocity potentials in the constant-depth regions on either side of the variable-depth region are represented analytically using appropriate eigenfunction expansions with unknown coefficients. For reviews of these ideas see Yeung (1975) and Liu \& Liggett (1982). The advantage of using a simpler Green's function has to be balanced against the need to discretize over extended boundaries. Nevertheless the method has been used to good effect and has enabled nonlinear as well as linear wave problems to be solved, including for example the propagation of solitary waves towards a beach from a region of constant depth (Kim, Liu \& Liggett 1983). More recently Chamberlain (1993) has used the mild-slope equation to solve a number of scattering problems for a variety of bottom profiles.

The simplest scattering problem where the depths on either side of the obstacle are different is the propagation of waves over a vertical step. This was considered by Miles (1967) using eigenfunction expansions in each of the separate regions of constant depth. By matching the potential and its first derivative across the common boundary, he was able to characterize the propagating waves on either side of the step in terms of the unknown horizontal velocity across the step which in turn was expressed as the solution of a singular integral equation of the first kind. By invoking a variational approximation he was able to obtain accurate results in good agreement, over a range of incident wavelengths, with the work of Newman (1965) who had considered the same problem when one of the depths was infinitely large. Mei \& Black (1969) used Miles' method to determine the scattering of a plane wave by a rectangular block on the bottom, by first exploiting symmetry.

Newman also considered the problem when the bottom profile joining regions of constant depth contains a long region also of (different) constant depth, and derives expressions for the reflection and transmission coefficients in terms of the corresponding coefficients for a plane wave incident on a bottom profile which is semi-infinite in extent. Ideas based on both Newman (1965) and Miles (1967) will play a key role in the present work.

Fitz-Gerald (1976) considers an arbitrary bottom profile in two dimensions and exploits complex-variable techniques to map the two-dimensional fluid regions into an infinite strip at the expense of a more complicated free-surface condition. This enables him to reduce the problem to the solution of an integro-differential equation from which he is able to prove uniqueness of the problem for general bottom profiles in the limits of wavelength either large or small compared to the transition width joining the unequal constant depths. He then develops an elaborate numerical scheme for solving for the reflection coefficient which gives fair agreement with the work of Mei \& Black (1969) on rectangular bottom profiles. He also provides results for a range of profiles all of which have known conformal mappings transforming the fluid region into an infinite strip. 
Hamilton (1977) also uses a conformal mapping which maps a general bottom profile in two dimensions into a strip so as to remove the possibilities of the depth function being non-analytic. In this way he is able to develop nonlinear long-wave equations whose zeroth-order approximation is much improved over a conventional treatment involving the actual bottom profile and which provides remarkably accurate results even for abrupt bottom topographies. The mapping technique has been used more recently by Nachbin \& Papanicolaou (1992b) in studying the reflection of waves in shallow channels by rapidly varying bottom topographies.

The main idea behind the present work stems from a paper by Devillard, Dunlop \& Souillard (1988) who, in extending the ideas of Anderson localization to water waves over random bottom profiles, have devised a transition matrix method for a class of arbitrary bottom shapes to solve the full linear reflection and transmission problem. They make two approximations to achieve this end. First they restrict the topographies that can be studied by assuming that the bottom profile can be discretized into a series of horizontal steps each of which has a horizontal length which is large compared to the local wavelength above it. This enables them to regard the local evanescent field generated at one shelf as negligible by the time it reaches the next. This wide-spacing approximation is effectively that used by Newman (1965) described above, and has been used by numerous authors in the water-wave context to good effect. See for example Evans (1990) and Martin (1984). Their second approximation is to utilize Miles' (1967) variational approximation to relate the propagating waves on either side of the shelf through an appropriate $2 \times 2$ transition matrix. This enables them to obtain the cumulative effect of all the steps on the reflection and transmission of an incident wave by multiplication of the transition matrices appropriate to each individual shelf. The idea has recently been taken up by O'Hare \& Davies (1992) who have shown that the method works well even when the transition matrix is simplified still further by using a plane-wave approximation for scattering at a step, suggested by Miles (1967). They use the method to consider the reflection by a series of sinusoidal bottom profiles and they are able to reproduce accurately the high refiection due to Bragg resonance when the wavelength is twice the wavelength of the bottom profile. Their results suggest that the method of Devillard et al. may have wider validity than expected. The use of stepped profiles to approximate general smooth bottom profiles has also been considered by Johnson (1990) in connection with the low-frequency scattering of Kelvin waves.

In the present paper we shall show how the idea of Devillard et al. (1988) can be adapted to deal with bottom profiles which are neither smooth nor low, in the sense that the horizontal extent of the corresponding discretized steps modelling the profile are large compared to the height of the steps. The idea is first to map the fluid regions into an infinite strip as described by Fitz-Gerald (1976) or Hamilton (1977) thereby transferring the difficulty associated with the variable geometry to one involving a variable boundary condition on the free surface. A discretization technique akin to that of Devillard et al. is then applied.

The advantages of performing this transformation are twofold. First, as explained by Hamilton, the effect of the mapping is to transform the equation of the bottom profile into a surface condition which varies more slowly with the horizontal coordinate. As an extreme example, a bottom profile consisting of an infinitely thin vertical barrier is transformed into a symmetric variable surface condition having a profile with a continuously varying tangent (Hamilton 1977, Figure 5). Because of the smoothing which has taken place we anticipate greater accuracy when discretizing this variable free-surface condition into piecewise constant sections than when discretizing 
the bottom profile directly.

There is however a much more important advantage in discretizing the free-surface condition rather than the bottom condition. Whereas Devillard et al. need the solution over a shelf given by Miles, which is expressed only approximately either in terms of a plane-wave or a variational approximation, the discretization of the freesurface condition requires the solution in a strip of constant width for the reflection and transmission of a wave incident from a semi-infinite region supporting waves of one wavenumber into a semi-infinite region supporting waves of a slightly different wavenumber. This problem has an explicit simple solution using the Wiener-Hopf technique which has been given by Weitz \& Keller (1950) who were concerned with modelling the propagation of waves into a region containing small pieces of floating ice which had the effect of modifying the free-surface condition. Equipped with this exact solution, which is rederived using a residue calculus method in an Appendix, we can, as in Devillard et al. (1988) construct a series of transition matrices carrying information about the propagating waves across the region of varying surface condition. Here too we shall assume the effect of the local evanescent modes from one junction is negligible at the next. The method is a natural extension to two dimensions of the idea of discretizing a one-dimensional wave equation having variable wavenumber and utilizing a sequence of transition matrices to obtain overall transmission and reflection properties. A good description is provided by Levine (1978, p.123). The only difference is that in the one-dimensional case the solution over each step of the discretization is exact and the process can be confidently expected to converge to the true solution as the size of each step is decreased, whereas the present application requires neglect of certain evanescent modes and we can be less confident of convergence to the true solution.

The plan of the paper is as follows. In $\$ 2$ a derivation is given of the transition matrix linking the progressive waves in two semi-infinite regions supporting waves of different wavenumbers. The derivation is entirely general at this stage since it is relevant to other situations also. For example, the different wavenumbers in each region might be due to the change in surface boundary condition arising from the mapping of the bottom profile as described above, or it might be due to a change in depth in passing over a shelf as in the original Miles (1967) paper. Again the governing equation could be the Helmholtz equation so that the difference in wavenumbers arose from a step change in an acoustic waveguide or from a sudden change in boundary conditions on the walls of a uniform guide.

In deriving the transition matrix, use is made of general reciprocity conditions linking the left and right reflection and transmission coefficients so that the transition matrix reduces finally to the product of four separate $2 \times 2$ matrices: three rotation matrices involving 'angles' related to the relative size of the particular shelf, and the phases of a left and right reflection coefficient, and a matrix involving the modulus of a left reflection coefficient and the ratio of successive wavenumbers.

Some examples of the application of the method are described in $\S 3$. In particular it is shown how the formulation of Devillard et al. can be retrieved.

In $\S 4$ we introduce our new technique for the solution of wave propagation over an arbitrary bottom topography in two dimensions. It is shown how the problem can be reduced to a problem in a uniform strip with a variable free-surface condition. This new problem is then discretized, and the transition matrix formulation of $\S 2$ applied, resulting in a simple scheme for the determination of the reflection and transmission coefficients.

In $\S 5$ the method is applied to the explicit simple solution derived by Roseau 
(1976) when the bottom profile joins smoothly two regions of unequal depth. A comparison is made between the Devillard approach and the discretized free-surface approach and it is shown that in this case the latter is superior in approximating the exact answer for the modulus of the reflection coefficient. Two other examples, where solutions can be computed using alternative methods, are used to validate the method. These are the scattering of waves by a single vertical step, and by a vertical barrier attached to the sea bed and extending part way to the free surface. In each case the transition matrix method proves to be simpler to implement compared to eigenfunction expansion methods, and also produces accurate results. The submerged barrier problem, which cannot be tackled by the method of Devillard et al., and which requires many terms in an eigenfunction expansion approach, is a particularly severe test of the present method, yet good accuracy is achieved.

\section{Transition matrix formulation}

In this section we shall be concerned with wave propagation in a two-dimensional region described by coordinates $x, y$, in which waves of wavenumber $k_{i}\left(k_{i+1}\right)$ exist for all $x$ with $x<x_{i}\left(>x_{i}\right)$, where $k_{i} \neq k_{i+1}$. The difference in wavenumber could arise, for example, from a sudden change in the constant width of the region defined by the coordinate $y$, or in a sudden change in the boundary condition on $y=0$, in each case as $x$ passes through $x_{i}$. Two distinct types of solution can be considered describing waves incident from either $x=-\infty$ or $x=+\infty$ and being partially reflected and partially transmitted at $x=x_{i}$.

Thus in the first case, for $x \rightarrow-\infty$ we may write

$$
\phi(x, y) \sim\left(\mathrm{e}^{\mathrm{i} k_{i} x}+R_{i} \mathrm{e}^{-\mathrm{i} k_{i} x}\right) \chi_{i}(y),
$$

whilst as $x \rightarrow+\infty$

$$
\phi(x, y) \sim T_{i} \mathrm{e}^{i k_{i+1} x} \chi_{i+1}(y) .
$$

For the latter case, as $x \rightarrow-\infty$

$$
\psi(x, y) \sim t_{i} \mathrm{e}^{-\mathrm{i} k_{i} x} \chi_{i}(y),
$$

whilst as $x \rightarrow+\infty$

$$
\psi(x, y) \sim\left(\mathrm{e}^{-\mathrm{i} k_{i+1} x}+r_{i} \mathrm{e}^{\mathrm{i} k_{i+1} x}\right) \chi_{i+1}(y) .
$$

Here $\chi_{i}$ is an appropriate eigenfunction for the governing equation and boundary conditions satisfying

$$
\int_{L} \chi_{j}^{2}(y) \mathrm{d} y=1, \quad j=i, i+1,
$$

where $L$ is the width of the region, which may be constant for all $x$ or may change abruptly from one constant value to another as $x$ passes through $x_{i}$. The quantities $R_{i}, r_{i}$ and $T_{i}, t_{i}$ are the reflection and transmission coefficients for the problem. Owing to the normalization condition (2.5), these may not correspond to the usual definitions of such coefficients.

Provided $\phi, \psi$ both satisfy either Laplace's equation, or a Helmholtz or modified Helmholtz equation, and on the infinite boundaries of the region both $\phi$ and $\psi$ satisfy the same conditions which may be Neumann, Dirichlet or a mixed condition, then it follows from Green's second identity that

$$
\int_{C}\left(\phi \frac{\partial \psi}{\partial n}-\psi \frac{\partial \phi}{\partial n}\right) \mathrm{d} s=0
$$


where $C$ is a closed contour bounding the region. It is clear that the only contribution to this integral arises from the lines $x= \pm X, X$ large, where the forms (2.1) to (2.4) apply.

It follows from (2.6) by direct calculation, that

$$
\alpha_{i} t_{i}=T_{i},
$$

where $\alpha_{i}=k_{i} / k_{i+1}$. Further relations are obtained by choosing $\phi$ and $\bar{\phi}, \psi$ and $\bar{\psi}$, and $\phi$ and $\bar{\psi}$ in turn, in place of $\phi, \psi$ in (2.6). In this way it can be shown that

$$
\begin{gathered}
\alpha_{i}\left(1-\left|R_{i}\right|^{2}\right)=\left|T_{i}\right|^{2}, \\
\left(1-\left|r_{i}\right|^{2}\right)=\alpha_{i}\left|t_{i}\right|^{2}
\end{gathered}
$$

and

$$
\alpha_{i} R_{i} \bar{t}_{i}+\overline{r_{i}} T_{i}=0,
$$

where a bar denotes complex conjugate.

It follows immediately from $(2.7)-(2.10)$ that

$$
\begin{gathered}
\left|R_{i}\right|=\left|r_{i}\right|, \\
T_{i} t_{i}-R_{i} r_{i}=T_{i} / \overline{T_{i}}=t_{i} / \bar{t}_{i}
\end{gathered}
$$

and

$$
R_{i} / t_{i}=-\bar{r}_{i} / \bar{t}_{i}
$$

For later use, we define real quantities $\rho_{i}, \theta_{i}, \Theta_{i}$ by

$$
r_{i} \mathrm{e}^{2 i k_{i+1} x_{i}}=\rho_{i} \mathrm{e}^{2 i \theta_{i}}
$$

and

$$
R_{i} \mathrm{e}^{-2 \mathrm{i} k_{i} x_{i}}=-\rho_{i} \mathrm{e}^{2 \mathrm{i} \Theta_{i}},
$$

$0<\rho_{i}<1$, which takes into account the fact that the reflection coefficients are with respect to the point $x=x_{i}$. It follows from equations (2.13) to (2.15) that we may define

$$
t_{i}=\tau_{i} \mathrm{e}^{\mathrm{i}\left(\theta_{i}+\theta_{i}\right)} \mathrm{e}^{-\mathrm{i}\left(k_{i+1}-k_{i}\right) x_{i}},
$$

where

$$
\tau_{i}=\left[\left(1-\rho_{i}^{2}\right) / \alpha_{i}\right]^{1 / 2} .
$$

We can generalize these particular two solutions by assuming that

$$
\Phi(x, y) \sim\left(A_{i} \mathrm{e}^{\mathrm{i} k_{i} x}+B_{i} \mathrm{e}^{-\mathrm{i} k_{i} x}\right) \chi_{i}(y) \quad \text { as } \quad x \rightarrow-\infty,
$$

and that

$$
\Phi(x, y) \sim\left(A_{i+1} \mathrm{e}^{i k_{i+1} x}+B_{i+1} \mathrm{e}^{-i k_{i+1} x}\right) \chi_{i+1}(y) \text { as } x \rightarrow+\infty,
$$

and the values of $A_{i+1}, B_{i+1}$ can be related to $A_{i}, B_{i}$ through the original particular solutions $\phi$ and $\psi$.

Thus we write

$$
\left(\begin{array}{l}
A_{i+1} \\
B_{i+1}
\end{array}\right)=S_{i}\left(\begin{array}{l}
A_{i} \\
B_{i}
\end{array}\right)
$$

and note that two special solutions are obtained by choosing

$$
\begin{aligned}
& \text { (i) } \left.\quad A_{i}=1, \quad B_{i}=R_{i}, \quad A_{i+1}=T_{i}, \quad B_{i+1}=0,\right\} \\
& \text { (ii) } \left.\quad A_{i}=0, \quad B_{i}=t_{i}, \quad A_{i+1}=r_{i}, \quad B_{i+1}=1 .\right\}
\end{aligned}
$$


Using these as column vectors in $(2.20)$ gives

$$
\left(\begin{array}{cc}
T_{i} & r_{i} \\
0 & 1
\end{array}\right)=S_{i}\left(\begin{array}{cc}
1 & 0 \\
R_{i} & t_{i}
\end{array}\right)
$$

whence

$$
\mathbf{S}_{i}=\left(\begin{array}{cc}
{\overline{t_{i}}}^{-1} & r_{i} t_{i}^{-1} \\
\overline{r_{i}} \bar{t}_{i}^{-1} & t_{i}^{-1}
\end{array}\right),
$$

where (2.12) and (2.13) have been used.

An alternative expression for $S_{i}$ can be obtained if use is made of (2.14) and (2.16). Thus we can write

$$
\boldsymbol{S}_{i}=\tau_{i}^{-1}\left(\begin{array}{cc}
\mathrm{e}^{-\mathrm{i} \boldsymbol{k}_{l+1} x_{i}} \mathrm{e}^{\mathrm{i} \theta_{l}} & 0 \\
0 & \mathrm{e}^{\mathrm{i} k_{i+1} x_{i}} \mathrm{e}^{-\mathrm{i} \theta_{i}}
\end{array}\right)\left(\begin{array}{cc}
1 & \rho_{i} \\
\rho_{i} & 1
\end{array}\right)\left(\begin{array}{cc}
\mathrm{e}^{\mathrm{i} k_{i} x_{i}} \mathrm{e}^{\mathrm{i} \theta_{i}} & 0 \\
0 & \mathrm{e}^{-i \boldsymbol{k}_{i} x_{i}} \mathrm{e}^{-\mathrm{i} \boldsymbol{\theta}_{i}}
\end{array}\right),
$$

from whence it follows that

$$
\left(\begin{array}{l}
A_{i+1} \mathrm{e}^{i k_{i+1} x_{i}} \mathrm{e}^{-\mathrm{i} \theta_{i}} \\
\boldsymbol{B}_{i+1} \mathrm{e}^{-i k_{i+1} x_{i}} \mathrm{e}^{\mathrm{i} \theta_{i}}
\end{array}\right)=\tau_{i}^{-1}\left(\begin{array}{cc}
1 & \rho_{i} \\
\rho_{i} & 1
\end{array}\right)\left(\begin{array}{c}
A_{i} \mathrm{e}^{\mathrm{i} k_{i} x_{i}} \mathrm{e}^{\mathrm{i} \theta_{i}} \\
B_{i} \mathrm{e}^{-\mathrm{i} k_{i} x_{i}} \mathrm{e}^{-\mathrm{i} \theta_{i}}
\end{array}\right) .
$$

We now define

$$
\begin{gathered}
\Psi_{i}=\left(A_{i} \mathrm{e}^{\mathrm{i} k_{i} x_{i}}+B_{i} \mathrm{e}^{-\mathrm{i} k_{i} x_{i}}\right), \\
\Omega_{i}=-k_{i}^{-1} \Psi_{i}^{\prime}=-\mathrm{i}\left(A_{i} \mathrm{e}^{\mathrm{i} k_{1} x_{i}}-B_{i} \mathrm{e}^{-\mathrm{i} k_{i} x_{i}}\right) .
\end{gathered}
$$

Note that, apart from the variation in $y, \Psi_{i}$ is the far-field potential in $x<x_{i}$ evaluated at $x=x_{i}$, whilst $\Psi_{i+1}$ is the far-field potential in $x>x_{i}$ evaluated at $x=x_{i+1}$. Note also that these definitions differ from the corresponding ones given in Devillard et al. (1988) and O'Hare \& Davies (1992) by the factor $\chi_{i}(0)$. After some algebra, it can be shown, starting from (2.25), that

$$
\left(\begin{array}{l}
\Psi_{i+1} \\
\boldsymbol{\Omega}_{i+1}
\end{array}\right)=\boldsymbol{R}_{\delta_{i}} \boldsymbol{R}_{\theta_{1}} \boldsymbol{M}_{i} \boldsymbol{R}_{\theta_{i}}\left(\begin{array}{l}
\Psi_{i} \\
\boldsymbol{\Omega}_{i}
\end{array}\right)=\boldsymbol{P}_{i}\left(\begin{array}{c}
\Psi_{i} \\
\Omega_{i}
\end{array}\right)
$$

say, where

$$
\boldsymbol{R}_{\delta}=\left(\begin{array}{cc}
\cos \delta & -\sin \delta \\
\sin \delta & \cos \delta
\end{array}\right)
$$

is a rotation matrix,

$$
\delta_{i}=k_{i+1}\left(x_{i+1}-x_{i}\right)
$$

and

$$
\boldsymbol{M}_{i}=\left(\begin{array}{cc}
\left(1+\rho_{i}\right) / \tau_{i} & 0 \\
0 & \left(1-\rho_{i}\right) / \tau_{i}
\end{array}\right)
$$

which depends solely on the magnitude of the reflection and transmission coefficients for a wave incident from $x>x_{i}$. Using (2.17) we obtain an alternative form for this matrix:

where

$$
\boldsymbol{M}_{i}=\alpha_{i}^{1 / 2}\left(\begin{array}{cc}
\gamma_{i} & 0 \\
0 & \gamma_{i}^{-1}
\end{array}\right)
$$

$$
\gamma_{i}=\left(\frac{1+\rho_{i}}{1-\rho_{i}}\right)^{1 / 2} .
$$

Equation (2.28) relates the values of the potential and its derivative at the junction 
point $x_{i}$ with the corresponding values at the next junction point $x_{i+1}$. Implicit in the derivation of (2.28) is the assumption that any local evanescent wave field existing in the vicinity of $x_{i}$ is negligible in its effect at $x_{i+1}$. With this assumption, (2.28) can be used repeatedly to connect $N$ regions of different constant wavenumbers through a single $2 \times 2$ matrix $\prod_{i=N-1}^{0} \boldsymbol{P}_{i}$ made up from matrices $\boldsymbol{M}_{i}$ and $\boldsymbol{R}_{\theta_{i}}, \boldsymbol{R}_{\theta_{i}}$ related to the amplitude and phase respectively of the reflection and transmission coefficients for waves incident upon a single junction at $x=x_{i}$, and from matrices $\boldsymbol{R}_{\delta_{i}}$ which measure the size of each region of constant wavelength. Note that, in contrast to $\boldsymbol{S}_{i}$ in (2.20), the $2 \times 2$ matrices relating $\Psi_{i}$ and its derivative to $\Psi_{i+1}$ in (2.28), are all real. Equation (2.28) is a new result and applies generally whenever there is a change in wavenumber at each point $x_{i}$ from $k_{i}$ to $k_{i+1}$ for whatever reason.

In the next section we shall consider specific examples of this idea in one or two space dimensions.

\section{Examples}

\section{A stretched string of variable density}

As a first example we consider the case of a stretched string of tension $T$ and of variable density $\rho(x)$ whose displacement $u(x, t)=\operatorname{Re}\left\{v(x) \mathrm{e}^{-\mathrm{i} \omega t}\right\}$ is described by

$$
\frac{\mathrm{d}^{2} v}{\mathrm{~d} x^{2}}+k^{2}(x) v=0, \quad-\infty<x<\infty,
$$

where $k(x)=\omega(\rho(x) / T)^{1 / 2}$. This problem is one-dimensional but the theory of $\S 2$ is applicable if the eigenfunctions $\chi_{j}(y)$ are chosen to be unity. Let $k(x)$ be discretized by

$$
k(x)=k_{i}, \quad x_{i-1}<x<x_{i}, i=1,2, \ldots, N+1,
$$

where $x_{0}$ and $x_{N+1}$ are used to represent $-\infty$ and $+\infty$ respectively. In order to apply the theory in $\S 2$, we require the reflection properties across a single junction $x_{i}$ at which point the wavenumber changes from $k_{i}$ to $k_{i+1}$. These are easily obtained by matching $v$ and $v^{\prime}$ at $x=x_{i}$, whence $\boldsymbol{R}_{\theta_{i}}=\boldsymbol{R}_{\theta_{i}}=I$, the identity matrix,

$$
\boldsymbol{M}_{i}=\left(\begin{array}{cc}
1 & 0 \\
0 & k_{i} / k_{i+1}
\end{array}\right)
$$

and

$$
\left(\begin{array}{l}
\Psi_{i+1} \\
\boldsymbol{\Omega}_{i+1}
\end{array}\right)=\boldsymbol{R}_{\delta_{i}} \boldsymbol{M}_{i}\left(\begin{array}{l}
\Psi_{i} \\
\boldsymbol{\Omega}_{i}
\end{array}\right), \quad i=1, \ldots, N-1 .
$$

We can now obtain an approximate solution for the scattering by an arbitrary $k^{2}(x)$ by repeated use of (3.4) to obtain the overall scattering properties in terms of products of $2 \times 2$ matrices. The above procedure for such one-dimensional problems has been discussed by many authors including Levine (1978). The method is particularly suited to the determination of the eigenvalues or vibrational frequencies of a finite string for general $k^{2}(x)$. For example, successive use of (3.4) to model a finite string by $N-1$ separate regions on each of which $k(x)$ is assumed constant relates the values of $\Psi_{i}, \Omega_{i}$ at either end of the string in terms of a $2 \times 2$ matrix. Then the condition of vanishing displacement at both ends is $\Psi_{1}=\Psi_{N}=0$ which in turn requires the top right-hand element of this matrix to vanish. It is this requirement which determines the eigenvalues. Computations for specific $k(x)$ for which the eigenvalues are known suggest that accurate approximations can be obtained by taking $N \geqslant 100$. 
The result (3.4) for the infinite string agrees with that developed by Levine (1978) using a slightly different notation. Levine shows that as $N$ is increased indefinitely the original equation (3.1) is recovered showing that the method will yield the exact result in this limit.

\section{Linear shallow water waves over an arbitrary bottom topography}

Another one-dimensional example is provided by the propagation of linear shallow water waves over an arbitrary bottom topography. The surface displacement $\operatorname{Re}\left\{u(x) e^{-i \omega t}\right\}$ of such waves satisfies (Lamb 1932, § 185)

$$
\frac{\mathrm{d}}{\mathrm{d} x}\left(h(x) \frac{\mathrm{d} u}{\mathrm{~d} x}\right)+k^{2} u=0,
$$

where $k^{2}=\omega^{2} / g$ and $h(x)$ is the shape of the bottom topography assumed to be constant in the $z$-direction.

Explicit solutions of (3.5) exist for special $h(x)$ only. Discretization of $h(x)$ leads to wavenumbers $k_{i}$, where

$$
k_{i}^{2}=k^{2} / h\left(x_{i}\right) \text { for } \quad x_{i-1}<x<x_{i},
$$

and $i=1,2, \ldots, N$, say. In order to apply (2.28) we need $\rho_{i}, \theta_{i}, \Theta_{i}$ for a change from $k_{i}$ to $k_{i+1}$ at $x=x_{i}$, in this case corresponding to a change in depth from $h_{i}=h\left(x_{i}\right)$ to $h_{i+1}=h\left(x_{i+1}\right)$. After applying continuity of displacement and mass flux across $x=x_{i}$, we obtain $\rho_{i}=\left(k_{i}-k_{i+1}\right) /\left(k_{i}+k_{i+1}\right), \theta_{i}=\Theta_{i}=0$ so that again $\boldsymbol{R}_{\theta_{i}}=\boldsymbol{R}_{\theta_{i}}=I$, but now

$$
\mathbf{M}_{i}=\left(\begin{array}{cc}
k_{i} / k_{i+1} & 0 \\
0 & 1
\end{array}\right)=\left(\begin{array}{cc}
\left(h_{i+1} / h_{i}\right)^{1 / 2} & 0 \\
0 & 1
\end{array}\right)
$$

The method now proceeds as before with $\chi_{j}(y)$ again equal to unity.

It should be noted that equation (3.5) can be transformed into an equation of the form (3.1) but there appears little advantage in doing this.

\section{Reflection of water waves by an arbitrary bottom topography}

This application was first considered by Devillard et al. (1988) in connection with extending ideas on Anderson localization to water waves. The smoothly varying topography is replaced by a series of horizontal shelves above each of which propagating waves exist, having wavelength based on the local water depth. The result (2.28) is directly applicable to this case. However, in order to exploit the method usefully a further approximation is necessary to estimate the required values of $\rho_{i}, \theta_{i}, \Theta_{i}$, which are determined from the scattering of an incident wave at a single vertical step at $x=x_{i}$. Devillard $e t$ al. utilize a variational approximation to these scattering coefficients developed by Miles (1967). O'Hare \& Davies (1992), by comparing both this approximation and a simpler plane-wave approximation with a full numerical solution obtained from including up to ten non-propagating modes, conclude that good agreement is obtained for small changes of depth relative to the overall depth, even when the horizontal length of each shelf is also small. The result (2.28) is shown in Appendix A to be in complete agreement with Devillard et al. once the appropriate variational or plane-wave approximation is used. This problem is considered using a new approach in the next section.

\section{Acoustic waveguides with variable boundary conditions}

As a further illustration of the technique we consider a two-dimensional acoustical waveguide of width $2 d$, on whose boundaries $(y= \pm d,-\infty<x<\infty)$ a variable 
impedance condition depending on $x$ is satisfied. Such conditions have been assumed in modelling the effect of various liners in absorbing sound as it travels down the duct. See for example Namba \& Fukushige (1980). Thus it is assumed that the time-harmonic displacement or pressure satisfies

$$
\phi_{y}-K(x) \phi=0, \quad y= \pm d,-\infty<x<\infty,
$$

whilst for $|y| \leqslant d,-\infty<x<\infty$,

$$
\phi_{x x}+\phi_{y y}+k^{2} \phi=0 .
$$

Here $k=\omega / c$, where $c$ is the speed of sound. The only requirement on $K(x)$ is that when replaced by its discretized equivalent, $K_{i}=K\left(x_{i}\right), x_{i-1}<x<x_{i}$, resulting solutions of (3.9) admit wavenumbers $\pm k_{i}$ supporting just a single travelling wave in each region $x_{i-1}<x<x_{i}$.

The advantage of the above method applied to this problem is that the fundamental problem, the reflection and transmission of acoustic waves at a junction $x_{i}$ in an infinite strip at which the wavenumber changes from $k_{i}$ to $k_{i+1}$, has an exact explicit solution, which can be derived using the Wiener-Hopf technique. This is in contrast to the previous example where the fundamental problem was the reflection and transmission across a shelf which does not have an exact, explicit solution, so that a further approximation is involved in using the technique for general bottom topographies.

The details of the acoustic problem will not be addressed here but will be considered in a later paper. However the advantages of working with explicit solutions in constant-width strips prompts us to reconsider the water-wave problem and seek to improve on the approach of Devillard et al. (1988). This is our main application of the transition matrix method and, since it forms the substance of this paper, merits a section to itself.

\section{Reflection over an arbitrary profile using intermediate mapping functions}

The essential idea of this section is first to follow the method of Fitz-Gerald (1976) to map the two-dimensional fluid region bounded by the mean free surface and the arbitrary bottom topography into a strip of constant width. Of course the disadvantage of this approach is that the usual linearized free-surface condition relating the potential and its normal derivative is replaced by a variable condition depending on the position along the strip. However, if this variable condition is discretized, so as to apply (2.28), it turns out that the problem which needs to be solved, namely the reflection at a junction $x_{i}$ joining two semi-infinite strips supporting different wavenumbers, has an exact analytical solution which has been derived using the Wiener-Hopf technique by Weitz \& Keller (1950). See also Keller \& Weitz (1953). Furthermore the transformation appears to convert rapid changes in depth to smoother variations in the free-surface condition which enables a wider range of bottom topographies, even discontinuities, to be considered (see Hamilton 1977).

We follow the notation of Fitz-Gerald closely. Thus we choose Cartesian coordinates $x, y$ with $y$ vertically upwards. Then the well-known equations governing linear time-harmonic water waves are described by a velocity potential

$$
\Phi(x, y, t)=\operatorname{Re}\left\{\phi(x, y) \mathrm{e}^{-\mathrm{i} \omega t}\right\}
$$

satisfying

$$
\phi_{x x}+\phi_{y y}=0, \quad-\infty<x<\infty,-b(x)<y<0
$$




$$
\begin{aligned}
\phi_{y}+b^{\prime}(x) \phi_{x} & =0, & y & =-b(x), \\
\phi_{y}-K \phi & =0, & y & =0 .
\end{aligned}
$$

Here $b(x)$ is the arbitrary bottom shape non-dimensionalized with respect to a depth $h$ and $K=\omega^{2} h / g$. We assume without loss of generality, that

$$
b(x) \rightarrow\left\{\begin{array}{ll}
1, & x \rightarrow-\infty \\
\epsilon, & x \rightarrow+\infty
\end{array},\right.
$$

where $\epsilon \in(0,1]$, and also that $b^{\prime}(x) \rightarrow 0,|x| \rightarrow \infty$. For large values of $x$ we assume

$$
\phi(x, y) \sim \frac{\cosh k_{-}(y+1)}{\cosh k_{-}}\left(\mathrm{e}^{i k_{-} x}+\widetilde{R} \mathrm{e}^{-i k_{-} x}\right), \quad x \rightarrow-\infty,
$$

whilst

$$
\phi(x, y) \sim \frac{\cosh k_{+}(y+\epsilon)}{\cosh k_{+} \epsilon} \widetilde{T} \mathrm{e}^{i k_{+} x}, \quad x \rightarrow+\infty .
$$

Here

$$
K=k_{-} \tanh k_{-}=k_{+} \tanh k_{+} \epsilon
$$

and $k_{ \pm}$are real and positive. The quantities $\widetilde{R}$ and $\widetilde{T}$ are the usual reflection and transmission coefficients in water wave problems, defined to be the ratio of the amplitudes of the reflected and transmitted waves respectively to that of the incident wave.

There is no loss in generality in assuming transmission into the shallow region. The corresponding reflection and transmission coefficients for transmission from shallow to deep water can be obtained from (2.7) and (2.9).

We now map the strip $D:-\infty<x<\infty,-b(x)<y<0$ in the $z(=x+\mathrm{i} y)$ plane into the strip $\mathscr{D}:-\infty<\xi<\infty,-1<\eta<0$ of the $\zeta(=\xi+i \eta)$ plane using a conformal mapping $z=Q(\zeta)$ so that the points at infinity correspond, the $\xi$-axis maps to the free surface $y=0$ and the line $\eta=-1$ maps on to the bottom profile $y=-b(x)$. It will be assumed that

$$
z=Q(\zeta) \sim\left\{\begin{array}{ll}
\zeta, & \xi \rightarrow-\infty \\
\epsilon \zeta, & \xi \rightarrow+\infty
\end{array},\right.
$$

so that the far-field behaviour of the transformed potential $\psi(\xi, \eta)=\phi(x, y)$, where $x+i y=Q(\xi+i \eta)$, is

$$
\begin{gathered}
\psi(\xi, \eta) \sim \frac{\cosh \kappa_{-}(\eta+1)}{\cosh \kappa_{-}}\left(\mathrm{e}^{\mathrm{i} \kappa_{-} \xi}+\widetilde{R} \mathrm{e}^{-\mathrm{i} \kappa_{-} \xi}\right), \quad \xi \rightarrow-\infty, \\
\psi(\xi, \eta) \sim \frac{\cosh \kappa_{+}(\eta+1)}{\cosh \kappa_{+}} \widetilde{T} \mathrm{e}^{\mathrm{i} \kappa_{+} \xi}, \quad \xi \rightarrow+\infty,
\end{gathered}
$$

where

Also $\psi$ satisfies

$$
\kappa_{-}=k_{-}, \quad \kappa_{+}=\epsilon k_{+} .
$$

$$
\begin{gathered}
\psi_{\xi \xi}+\psi_{\eta \eta}=0, \quad-\infty<\xi<\infty,-1<\eta<0, \\
\psi_{\eta}=0, \quad \eta=-1, \\
\psi_{\eta}-K h(\xi) \psi=0, \quad \eta=0,
\end{gathered}
$$


where

$$
h(\xi)=\left.\frac{\mathrm{d} z}{\mathrm{~d} \zeta}\right|_{\eta=0} \sim\left\{\begin{array}{ll}
1, & \xi \rightarrow-\infty \\
\epsilon, & \xi \rightarrow+\infty
\end{array} .\right.
$$

Fitz-Gerald (1976) discusses methods for the determination of suitable approximations to $h(\xi)$ for given bottom profiles $b(x)$ as well as giving many examples of particular profiles. For the present we shall assume $h(\xi)(>0)$ is known and we shall discretize it in the form

$$
K_{i}=K h\left(\xi_{i}\right), \quad \xi_{i-1}<\xi<\xi_{i}, i=1,2, \ldots, N,
$$

so that the free-surface boundary condition is

$$
K_{i} \psi-\psi_{\eta}=0, \quad \xi_{i-1}<\xi<\xi_{i},
$$

which permits a travelling wave of wavenumber $k_{i}$ where, from (4.13), (4.14) and (4.18),

$$
K_{i}=k_{i} \tanh k_{i}
$$

defines $k_{i}$ and, from (2.5),

$$
\chi_{i}(\eta)=2 k_{i}^{1 / 2}\left(2 k_{i}+\sinh 2 k_{i}\right)^{-1 / 2} \cosh k_{i}(\eta+1) .
$$

The endpoints $\xi_{0}, \xi_{N}$ are such that $h\left(\xi_{0}\right) \approx 1$ and $h\left(\xi_{N}\right) \approx \epsilon$, the asymptotic values of $h(\xi)$. Then use of $(2.28)$ relates $\Psi_{N}, \Omega_{N}$ to $\Psi_{0}, \Omega_{0}$ through the $2 \times 2$ matrix $\prod_{i=N-1}^{0} P_{i}$. The canonical problem here is the solution of (4.13), (4.14) and

$$
\left.\begin{array}{rlrl}
K_{i} \psi-\psi_{\eta} & =0, & & \xi<\xi_{i}, \eta=0 \\
K_{i+1} \psi-\psi_{\eta} & =0, & \xi>\xi_{i}, \eta=0,
\end{array}\right\}
$$

which has been considered by Weitz \& Keller (1950) using the Wiener-Hopf technique. It is shown in Appendix B, using the simpler residue calculus method described by Mittra \& Lee (1971) that, in the notation of $\S 2$,

$$
\begin{aligned}
\rho_{i} & =\left(k_{i+1}-k_{i}\right) /\left(k_{i+1}+k_{i}\right), \\
\theta_{i} & =\sum_{n=1}^{\infty}\left\{\tan ^{-1}\left(k_{i+1} / \alpha_{n}^{i}\right)-\tan ^{-1}\left(k_{i+1} / \alpha_{n}^{i+1}\right)\right\}, \\
\Theta_{i} & =\sum_{n=1}^{\infty}\left\{\tan ^{-1}\left(k_{i} / \alpha_{n}^{i+1}\right)-\tan ^{-1}\left(k_{i} / \alpha_{n}^{i}\right)\right\},
\end{aligned}
$$

where $\alpha_{n}^{j}, j=i, i+1$, are the real positive roots of

$$
K_{j}+\alpha_{n}^{j} \tan \alpha_{n}^{j}=0 .
$$

The reflection and transmission coefficents can now be obtained in the following way. With $\rho_{i}, \theta_{i}, \Theta_{i}$ given by (4.22)-(4.24), $P_{i}, i=0, \ldots, N-1$ can be determined and $\Psi_{N}, \Omega_{N}$ related to $\Psi_{0}, \Omega_{0}$. This can easily be converted into a relationship between $A_{N}, B_{N}$ and $A_{0}, B_{0}$ by noting that, from (2.26) and (2.27), with $\xi_{i}$ replacing $x_{i}$,

$$
\left(\begin{array}{l}
\Psi_{i} \\
\Omega_{i}
\end{array}\right)=\left(\begin{array}{cc}
1 & 1 \\
-\mathrm{i} & \mathrm{i}
\end{array}\right)\left(\begin{array}{c}
A_{i} \mathrm{e}^{\mathrm{i} k_{i} \xi_{i}} \\
B_{i} \mathrm{e}^{-\mathrm{i} k_{i} \xi_{i}}
\end{array}\right) .
$$

Finally $A_{N+1}, B_{N+1}$ are related to $A_{N}, B_{N}$ through an application of (2.20) with $i=N$. We can thus compute the elements $c_{m n}$ of a $2 \times 2$ matrix $C$ such that

$$
\left(\begin{array}{l}
A_{N+1} \\
B_{N+1}
\end{array}\right)=\mathrm{C}\left(\begin{array}{l}
A_{0} \\
B_{0}
\end{array}\right)
$$


It can easily be shown that $\operatorname{det} C=\kappa_{-} / \kappa_{+}$. Putting $A_{0}=1, B_{0}=R, A_{N+1}=T$, $B_{N+1}=0$ shows that

$$
R=-c_{21} c_{22}^{-1}, \quad T=\kappa_{-}\left(\kappa_{+} c_{22}\right)^{-1} .
$$

The quantities $\widetilde{R}$ and $\widetilde{T}$ are readily shown to be related to $R$ and $T$ by

$$
\begin{gathered}
\widetilde{R}=R \\
\widetilde{T}=T \frac{\cosh \kappa_{+}}{\cosh \kappa_{-}} \frac{\left(1+\sinh 2 \kappa_{-} / 2 \kappa_{-}\right)^{1 / 2}}{\left(1+\sinh 2 \kappa_{+} / 2 \kappa_{+}\right)^{1 / 2}} .
\end{gathered}
$$

It should be pointed out that one can proceed directly to (4.27) by repeated application of (2.20) without requiring the introduction of the variables $\Psi_{i}$ and $\Omega_{i}$. This method does have the advantage of computational simplicity. However, as pointed out by Devillard et al. (1988) and O'Hare \& Davies (1992), the $\Psi_{i}, \Omega_{i}$ variables have more physical meaning, and also the formulation in terms of (2.28) is more elegant and it is easier to see the effects of the various contributions due to the width of the regions and the reflection and transmission coefficients for each canonical problem.

\section{Results}

\section{Roseau's explicit solution}

The only known explicit solution for the reflection coeffcient $|R|$ is that due to Roseau (1976) who considered a smooth monotonic transition from constant depth 1 to depth $\epsilon$. In the present notation, he used the mapping function

$$
z=Q(\zeta)=\zeta+\beta^{-1}(\epsilon-1) \ln \left(1+\mathrm{e}^{\beta \zeta}\right),
$$

where $\beta \in(0, \pi / 2)$, to map the fluid region into a strip of width 1 , when the function $h$ becomes

$$
h(\xi)=\left(1+\epsilon \mathrm{e}^{\beta \xi}\right) /\left(1+\mathrm{e}^{\beta \xi}\right) .
$$

The parameter $\beta$ allows for variations in the lengthscale of the transition from one depth to the other, see Fitz-Gerald (1976). Thus as $\beta \rightarrow \pi / 2$ the bottom profile becomes steeper whereas as $\beta \rightarrow 0$ the 'transition width' becomes infinite. In the physical plane the bottom is described by the curve

$$
\beta x=\ln \mu+\frac{1}{2}(\epsilon-1) \ln \left[1+2 \mu \cos \beta+\mu^{2}\right],
$$

where

$$
\mu=\lambda(\sin \beta-\lambda \cos \beta)^{-1}, \quad \lambda=\tan [\beta(y+1) /(1-\epsilon)] .
$$

Roseau was then able to solve the resulting boundary-value problem in the strip with the variable boundary condition (4.15) with $h$ given by (5.2) by solving an equivalent functional difference equation. A re-working and extension of the method is given in Evans (1985). He obtained the explicit form

$$
|\widetilde{R}|=\left|\frac{\sinh \pi \beta^{-1}\left(k_{-}-\epsilon k_{+}\right)}{\sinh \pi \beta^{-1}\left(k_{-}+\epsilon k_{+}\right)}\right|,
$$

where $k_{ \pm}$satisfy (4.8). The transmission coefficient, $|\widetilde{T}|$ can then be computed from (2.8) and (4.30).

As a first test of the theory developed in $\S 2$ we shall discretize the surface condition 


\begin{tabular}{ccccccccc}
$K, \epsilon, \beta=$ & \multicolumn{2}{c}{$1,0.5,1$} & & \multicolumn{2}{c}{$0.1,0.1,1.5$} & & \multicolumn{2}{c}{$0.1,0.1,0.1$} \\
$N$ & $|\widetilde{R}|$ & $|\widetilde{T}|$ & & $|\widetilde{R}|$ & $|\widetilde{T}|$ & & $|\widetilde{R}|$ & $|\widetilde{T}|$ \\
10 & 0.1561 & 1.0336 & & 0.4729 & 1.5318 & & 0.3939 & 1.5980 \\
20 & 0.0060 & 1.0464 & & 0.4776 & 1.5274 & & 0.1932 & 1.7057 \\
50 & 0.0072 & 1.0464 & & 0.4789 & 1.5261 & & 0.0019 & 1.7385 \\
100 & 0.0073 & 1.0464 & & 0.4791 & 1.5259 & & 0.0019 & 1.7385 \\
& & & & & & & & \\
Exact & 0.0073 & 1.0464 & & 0.4792 & 1.5258 & & 0.0018 & 1.7385 \\
Devillard & 0.0096 & 1.0463 & & 0.4788 & 1.5262 & & 0.0057 & 1.7385
\end{tabular}

TABLE 1. Comparison with the exact solution of Roseau and the method of Devillard et al.

(5.2) as described in $\$ 4$. In this example, and throughout this section, we shall only use equal spacing, i.e. $\xi_{i+1}-\xi_{i}$ will be kept constant. For any particular application this is unlikely to be the most efficient choice. However, as we shall see, it is perfectly adequate and has the merit of great simplicity. Thus for a given $K, \epsilon$ and $\beta$ we must choose $N, \xi_{0}$ and $\xi_{N}$. In this case we take $\xi_{0}=-\xi_{N}$ and choose $\xi_{0}$ such that $\left|K_{0}-K\right|<10^{-6}$. This condition ensures that spurious reflections do not occur near the endpoints, but of course does mean, given the choice of equal spacing, that in situations where the transition width is very wide, large values of $N$ will be required even though the slope of the bottom profile is very mild. For computational purposes, it is also necessary to truncate the infinite sums (4.23) and (4.24) but it was found that only very few terms were required. In the calculations below 10 terms were used.

To fix ideas we consider three specific examples, namely $(K, \epsilon, \beta)=(1,0.5,1)$, $(0.1,0.1,1.5)$ and $(0.1,0.1,0.1)$. The results are shown in table 1 . In the first example the parameters are chosen to have fairly moderate values, whereas in the other two examples the waves are chosen to be very long so as to maximize the effect of the bottom topography. In the second example the transition width is very small whilst in the final example it is extremely large. The values of $\xi_{N}$ chosen to ensure that the difference between $K_{0}$ and $K$ is less than $10^{-6}$ are approximately 6.6, 3.8 and 57.0 respectively.

A comparison of our results with the method of Devillard et al. (1988) is also shown in table 1. The form of the bottom profile as given by equations (5.3) and (5.4) is not convenient for discretization in $x$ and so in this case discretization in equal intervals of $y$ has been carried out. The convergence characteristics of the Devillard approach do not appear to be as good as those for our method. It was found, by trial and error, that satisfactory numerical results could be obtained by using a discretization parameter of $N=200$ and taking 40 terms in the sum (A 4).

The table clearly demonstrates the power of the intermediate mapping function method.

\section{The single step}

Fitz-Gerald (1976) gives a mapping function corresponding to 'mounds superimposed on steps' which constitutes another family of bottom profiles with different constant depths at $x= \pm \infty$. A special case of this mapping which can be used to provide further validity to our method is that of a single step. If the depths at $-\infty$ and $+\infty$ are again 1 and $\epsilon$ respectively, the function $h$ is given by

$$
h(\xi)=\left(1+\epsilon^{2} \mathrm{e}^{\pi \xi}\right)^{1 / 2} /\left(1+\mathrm{e}^{\pi \xi}\right)^{1 / 2} .
$$




\begin{tabular}{|c|c|c|c|c|c|c|}
\hline \multirow{3}{*}{$\begin{array}{c}K, \epsilon= \\
N \\
10\end{array}$} & \multicolumn{2}{|c|}{$1,0.5$} & \multicolumn{2}{|c|}{$1,0.1$} & \multicolumn{2}{|c|}{$0.1,0.1$} \\
\hline & $|\widetilde{\boldsymbol{R}}|$ & $|\widetilde{T}|$ & $|\widetilde{R}|$ & $|\widetilde{T}|$ & $|\vec{R}|$ & $|\widetilde{T}|$ \\
\hline & 0.0629 & 1.0443 & 0.3816 & 1.3054 & 0.5042 & 1.5013 \\
\hline 20 & 0.1091 & 1.0411 & 0.4016 & 1.2934 & 0.5090 & 1.4964 \\
\hline 50 & 0.1208 & 1.0387 & 0.4168 & 1.2838 & 0.5101 & 1.4953 \\
\hline 100 & 0.1223 & 1.0385 & 0.4192 & 1.2823 & 0.5103 & 1.4951 \\
\hline Eigenfunction & 0.1113 & 1.0399 & 0.4132 & 1.2861 & 0.5098 & 1.4956 \\
\hline Variational & 0.1205 & 1.0388 & 0.4196 & 1.2820 & 0.5104 & 1.4950 \\
\hline Plane-wave & 0.0859 & 1.0425 & 0.3454 & 1.3254 & 0.5039 & 1.5016 \\
\hline
\end{tabular}

TABLE 2. Reflection by a single step

Reflection and transmission coefficients calculated using the intermediate mapping technique, using the same truncation parameters as for the Roseau profiles, are shown in table 2 for the three different pairs of values of $K$ and $\epsilon$. For comparison, values computed using three alternative methods are also shown. These are the method of matched eigenfunction expansions together with the variational method and the plane-wave approximation due to Miles (1967). None of the results in the table can be considered exact, but provided sufficiently large truncation sizes are used the matched eigenfunction method should be capable of arbitrary accuracy. In the table a truncation parameter of $N=80$ was used. Tests with higher values of $N$ show that these results are accurate to at least 3 decimal places and owing to the nature of the variational approximation (here 80 terms were taken in the infinite sums computed, see Appendix A) the exact answer will lie between the result from the eigenfunction method and that from the variational method.

Again the table shows clearly how accurate values for the reflection and transmission coefficients can be obtained with very little computational effort using our technique.

\section{The vertical barrier}

In both of the previous examples the method of Devillard et al. can be used - the single step is of course a trivial application. Our final example is of a case that is not amenable to their treatment, that of a vertical barrier attached to the sea bed, extending part way to the free surface. In infinitely deep water this problem has an explicit soluion due to Ursell (1947) who showed that

$$
|T|=\pi I_{0}(K b)\left[\pi^{2} I_{0}^{2}(K b)+K_{0}^{2}(K b)\right]^{1 / 2},
$$

where $b$ is the size of the gap above the barrier.

In finite water depth no such explicit solution is possible. Recently however $\mathbf{R}$. Porter (1994, personal communication) has obtained extremely accurate complementary bounds for $|R|$ and $|T|$ by using expansions in Tchebyshev polynomials for either the horizontal velocity across the gap or the jump in potential across the barrier, each of which appears as an unknown in a singular integral equation arising from an approach using matched eigenfunction expansions.

The geometry of the vertical barrier is a special case of a family of bottom profiles termed 'plateaus' by Fitz-Gerald (1976). If the water depth is 1 everywhere and the barrier has height $a(<1)$ then the function $h$ is given by

$$
h(\xi)=\cos (\pi a / 2)\left[1-\sin ^{2}(\pi a / 2) \tanh ^{2}(\xi \pi / 2)\right]^{1 / 2} .
$$




\begin{tabular}{|c|c|c|c|c|c|c|}
\hline$K, a=$ & \multicolumn{2}{|c|}{$1,0.5$} & \multicolumn{2}{|c|}{$0.1,0.5$} & \multicolumn{2}{|c|}{$1,0.9$} \\
\hline$N$ & $|\widetilde{R}|$ & $|\widetilde{T}|$ & $|\widetilde{\boldsymbol{R}}|$ & $|\widetilde{T}|$ & $|\widetilde{R}|$ & $|\widetilde{T}|$ \\
\hline 10 & 0.1370 & 0.9906 & 0.0672 & 0.9977 & 0.5560 & 0.8312 \\
\hline 20 & 0.1475 & 0.9891 & 0.0677 & 0.9977 & 0.5916 & 0.8063 \\
\hline 50 & 0.1523 & 0.9883 & 0.0679 & 0.9977 & 0.6028 & 0.7979 \\
\hline 100 & 0.1529 & 0.9882 & 0.0679 & 0.9977 & 0.6042 & 0.7968 \\
\hline Porter & 0.1533 & 0.9882 & 0.0680 & 0.9977 & 0.6054 & 0.7959 \\
\hline
\end{tabular}

TABLE 3. Reflection by a vertical barrier

Discretization of this function and application of our transition matrix method provides results which are compared with Porter's 'exact' numerical results in table 3. Again excellent agreement is obtained.

\section{Conclusions}

We have presented a new method for the determination of the reflection and transmission coefficients for the two-dimensional problem of the scattering of an incident wave of small amplitude by a varying bottom topography. The first step of the method is to transform the fluid region into a strip of constant width and so in order for the technique to be useful a mapping must be found to perform this transformation. A number of such functions, suitable for a variety of families of bottom profiles, are given in Fitz-Gerald (1976) who also addresses the general problem of finding a mapping function which approximates to a given bottom topography. For example, by replacing $\xi$ by $\beta \xi, \beta \in(0,1]$ in the step and barrier cases described in the previous section he shows how a class of smooth bottom shapes can be generated which approach the step or barrier as $\beta \rightarrow 1$. A good general discussion on conformal mappings, including how to round off corners in Schwarz-Christoffel mappings is given in Henrici (1974). Once such a mapping has been found the method provides a very efficient technique for the evaluation of the reflection and transmission coefficients.

The fundamental idea behind our approach is to discretize the resulting boundaryvalue problem in the strip into a finite number of sections, each of which has constant boundary conditions on its boundaries. The idea of using discretization techniques to solve the water-wave scattering problem is not knew, recent work having been done on this topic by Devillard et al. (1988) and O'Hare \& Davies (1992). The approach used in their papers is to discretize the fluid domain into regions each of which has constant depth. Such a technique has the advantage that it is applicable to a wider class of geometries - since no mapping has to be found - but has the disadvantage that the canonical problem that must be solved for each region is the scattering by a single vertical step, for which no exact analytical solution exists. On the other hand an exact analytic solution to the canonical problem that arises when the boundary-value problem in the strip is discretized does exist. It is also worth noting that there are geometries which can be mapped into a strip for which the method of Devillard et al. cannot be used, the simplest of which is the vertical barrier considered in $\S 5$.

As a first step in the development of our new technique, we have formulated a transition matrix approach, applicable to a wide class of problems where the domain can be discretized into regions each supporting waves of a different wavenumber. We 
have applied this method to a number of alternative problems, both one- and twodimensional. Such problems include not only problems of reflection and transmission but also that of the determination of natural frequencies of vibration.

For the water-wave reflection problem, we have validated our method by comparing it with the only known explicit solution, due to Roseau (1976), and with two other examples where the reflection and transmission coefficients can be computed using alternative means. In all cases it was found that our intermediate mapping technique gave extremely accurate results with minimal computer effort.

Both the discretized bottom profile method due to Devillard et al. and the intermediate mapping and discretized free-surface condition method used here require the neglect of the evanescent modes from one junction to the next, and the validity of this approximation as the number of discretizations increases or as the bottom profile becomes steeper is open to question. Thus, according to a referee, although the Devillard method works surprisingly well for the sinusoidal bottom profile considered by O'Hare \& Davies (1992) it does not work for any rapidly varying bottom profiles. It is clear that much work needs to be done on both methods to investigate the precise conditions on the bottom profile or free-surface condition under which the neglect of the evanescent modes leads to an accurate approximation as the number of discretizations increases. Nevertheless the success of the present method in predicting $|T|$ for the extreme case of a submerged vertical barrier or sharp spike extending upwards from the bottom, for which the Devillard method fails completely, suggests that the method is capable of predicting accurate values of $|T|$ even for the most rapidly varying profiles, and is presumably less sensitive to the neglect of the evanescent modes.

The work of C.M.L. was supported in part by SERC under grant GR/F/83969

\section{Appendix A. The formulation of Devillard et al.}

Consider the scattering of a surface wave, incident from $x=+\infty$, by a step at $x=x_{i}$. For $x<x_{i}\left(>x_{i}\right)$ the depth of water is taken to be $h_{i}\left(h_{i+1}\right)$. No exact solution exists to this problem, but approximations to the reflection and transmission coefficients, $r_{i}$ and $t_{i}$, can be obtained using the variational formulation described in Miles (1967).

The following notation will be used. Where $<(>)$ appears as a subscript or superscript it represents $i$ if $h_{i}<h_{i+1}\left(h_{i}>h_{i+1}\right)$ and $i+1$ if $h_{i+1}<h_{i}\left(h_{i+1}>h_{i}\right)$. The reflection and transmission coefficients are given by

$$
\begin{aligned}
r_{i} & =\frac{k_{i} k_{i+1}+\mathrm{i} c X^{-1}\left(k_{i+1} c^{J}-k_{i} c^{-J}\right)}{k_{i} k_{i+1}+\mathrm{i} c X^{-1}\left(k_{i+1} c^{J}+k_{i} c^{-J}\right)} \mathrm{e}^{-2 i k_{i+1} x_{i}}, \\
t_{i} & =\frac{2 \mathrm{i} k_{i+1} c X^{-1}}{k_{i} k_{i+1}+\mathrm{i} c X^{-1}\left(k_{i+1} c^{J}+k_{i} c^{-J}\right)} \mathrm{e}^{\mathrm{i}\left(k_{i}-k_{i+1}\right) x_{i}} .
\end{aligned}
$$

Here

$$
\begin{aligned}
c & =\int_{-h_{<}}^{0} \chi_{i}(y) \chi_{i+1}(y) \mathrm{d} y \\
& =\frac{4\left(k_{i} k_{i+1}\right)^{1 / 2} k_{>} \sinh k_{>}\left|h_{i+1}-h_{i}\right|}{\left(2 k_{i} h_{i}+\sinh 2 k_{i} h_{i}\right)^{1 / 2}\left(2 k_{i+1} h_{i+1}+\sinh 2 k_{i+1} h_{i+1}\right)^{1 / 2}\left(k_{<}^{2}-k_{>}^{2}\right)},
\end{aligned}
$$




$$
X=\frac{16 k_{<}}{2 k_{<} h_{<}+\sinh 2 k_{<} h_{<}} \sum_{n=1}^{\infty} \frac{\left(\alpha_{n}^{>} \sin \alpha_{n}^{>}\left|h_{i+1}-h_{i}\right|\right)^{2}}{\left(2 \alpha_{n}^{>} h_{>}+\sin 2 \alpha_{n}^{>} h_{>}\right)\left(k_{<}^{2}+\alpha_{n}^{>2}\right)} ;
$$

$\alpha_{n}^{<}$and $\alpha_{n}^{>}$are the real positive roots of

$$
K+\alpha_{n}^{<} \tan \alpha_{n}^{<} h_{<}=K+\alpha_{n}^{>} \tan \alpha_{n}^{>} h_{>}=0, \quad K=\omega^{2} / g,
$$

and

$$
J=\left\{\begin{array}{lll}
-1 & \text { if } & h_{i}=h_{<} \\
+1 & \text { if } & h_{i}=h_{>}
\end{array} .\right.
$$

The quantities $\rho_{i}, \theta_{i}$ and $\Theta_{i}$ are easily calculated from (A 1) and (A 2), and if these are substituted into (2.28) we obtain

$$
\left(\begin{array}{l}
\Psi_{i+1} \\
\Omega_{i+1}
\end{array}\right)=\boldsymbol{R}_{\delta_{i}}\left(\begin{array}{cc}
c^{J} & -k_{i} X / c \\
0 & \left(k_{i} / k_{i+1}\right) c^{-J}
\end{array}\right)\left(\begin{array}{l}
\Psi_{i} \\
\Omega_{i}
\end{array}\right)
$$

This is equivalent to the form quoted by Devillard et al. (1988), taking into account the slightly different definitions of $\Psi_{i}$ and $\Omega_{i}$ that we have used. O'Hare \& Davies (1992) also use the plane-wave approximation discussed by Miles (1967). This is equivalent to setting $X=0$ in the above formula.

\section{Appendix B. The residue calculus method applied to the reflection and transmission of water waves by a change in free-surface boundary condition}

We wish to solve (4.13), (4.14) and (4.21) together with appropriate radiation conditions. For a number of reasons the notation of the main text is not convenient for this task and so in this Appendix we will use a different notation. We will solve the following boundary-value problem:

$$
\begin{aligned}
\phi_{x x}+\phi_{y y} & =0, & & -\infty<x<\infty,-1<y<0, \\
\phi_{y} & =0, & & -\infty<x<\infty, y=-1, \\
K_{1} \psi-\psi_{y} & =0, & & x<\xi, y=0, \\
K_{2} \psi-\psi_{y} & =0, & & x>\xi, y=0,
\end{aligned}
$$

and we will assume that a wave is incident from $x=-\infty$.

This problem has been considered (for $\xi=0$ ) by Weitz \& Keller (1950) and Keller $\&$ Weitz (1953) in connection with the reflection and transmission of water waves by floating ice. In fact they solve the more general problem where the equation to be satisfied in the fluid is a modified Helmholtz equation rather than Laplace's equation. The method they employ is the Wiener-Hopf technique. However, a solution to (B 1)(B 4) can be obtained in a much simpler manner by using the residue calculus method described by Mittra \& Lee (1971). In fact Mittra \& Lee (p.65 example 2-8) give an outline solution to a similar more general problem with an impedance condition also on $y=-1$ for all $x$, but where the governing equation is the Helmholtz equation.

We begin by defining the orthonormal eigenfunctions

$$
\psi_{n}^{(1)}(y)=N_{n}^{(1)} \cos k_{n}(y+1), \quad \psi_{n}^{(2)}(y)=N_{n}^{(2)} \cos \kappa_{n}(y+1), \quad n=0,1, \ldots,
$$

where

$$
N_{n}^{(1)}=2 k_{n}^{1 / 2}\left(2 k_{n}+\sin 2 k_{n}\right)^{-1 / 2}, \quad N_{n}^{(2)}=2 \kappa_{n}^{-1 / 2}\left(2 \kappa_{n}+\sin 2 \kappa_{n}\right)^{-1 / 2}
$$

Here $k_{n}, \kappa_{n}, n \geqslant 1$ are the real positive roots of $k_{n} \tan k_{n}+K_{1}=0$ and $\kappa_{n} \tan \kappa_{n}+K_{2}=0$ 
respectively, whilst $k_{0}=-\mathrm{i} k, \kappa_{0}=-\mathrm{i} \kappa, k$ and $\kappa$ being the real, positive roots of $k \tanh k=K_{1}$ and $\kappa \tanh \kappa=K_{2}$.

In $x<\xi(>\xi)$ we set $\phi=\phi_{1}\left(\phi_{2}\right)$, where

$$
\begin{aligned}
& \phi_{1}=\mathrm{e}^{\mathrm{i} k x} \psi_{0}^{(1)}(y)+\sum_{n=0}^{\infty} A_{n} \mathrm{e}^{k_{n}(x-\xi)} \psi_{n}^{(1)}(y), \\
& \phi_{2}=\sum_{n=0}^{\infty} B_{n} \mathrm{e}^{-\kappa_{n}(x-\xi)} \psi_{n}^{(2)}(y),
\end{aligned}
$$

for some unknown coefficients $A_{n}$ and $B_{n}$. The reflection and transmission coefficients $R$ and $T$ are then related to $A_{0}$ and $B_{0}$ through

$$
R=A_{0} \mathrm{e}^{\mathrm{i} k \xi}, \quad T=B_{0} \mathrm{e}^{-\mathrm{i} \kappa \xi} .
$$

Matching $\phi$ and $\partial \phi / \partial x$ at $x=\xi$, using the orthogonality of the depth eigenfunctions and then eliminating the coefficients $A_{n}$ leads to the infinite system of equations

$$
\sum_{n=0}^{\infty} \frac{V_{n}}{\kappa_{n}-k_{m}}=A \delta_{m 0}, \quad m=0,1, \ldots,
$$

where

$$
V_{n}=B_{n} N_{n}^{(2)} \cos \kappa_{n}
$$

and

$$
A=\frac{2 \mathrm{i} k \mathrm{e}^{\mathrm{ik} \xi}}{\left(K_{2}-K_{1}\right) N_{0}^{(1)} \cosh k}
$$

Consider

$$
I=\frac{1}{2 \pi \mathrm{i}} \int_{C_{N}} \frac{f(z)}{z-k_{m}} \mathrm{~d} z,
$$

where $f(z)$ has simple poles at $z=\kappa_{n}, n=0,1, \ldots$, simple zeros at $z=k_{n}, n=1,2, \ldots$, and $f(z)=O\left(|z|^{-1 / 2}\right)$ as $|z| \rightarrow \infty$ on $C_{N}$. Here $C_{N}$ is a sequence of circles whose radius $R_{N}$ increases without bound as $N \rightarrow \infty$ whilst avoiding the zeros of the integrand. The conditions on $f(z)$ are sufficient to ensure that $I_{m} \rightarrow 0$ as $N \rightarrow \infty$. If we further assume that $f\left(k_{0}\right)=-1$ we obtain

$$
\sum_{n=0}^{\infty} \frac{\operatorname{Res}\left(f ; \kappa_{n}\right)}{\kappa_{n}-k_{m}}=\delta_{m 0}, \quad m=0,1, \ldots,
$$

from which it follows that

$$
V_{n}=A \operatorname{Res}\left(f ; \kappa_{n}\right) .
$$

A suitable function $f$ is given by

$$
f(z)=-\frac{\left(1-k_{0} / \kappa_{0}\right)}{\left(1-z / \kappa_{0}\right)} \prod_{n=1}^{\infty} \frac{\left(1-z / k_{n}\right)\left(1-k_{0} / \kappa_{n}\right)}{\left(1-z / \kappa_{n}\right)\left(1-k_{0} / k_{n}\right)}
$$

It follows from (B 9), (B 11) and (B 15) that

$$
T=\frac{2 k P(\kappa-k) \mathrm{e}^{\mathrm{j}(k-\kappa) \xi}}{\left(K_{2}-K_{1}\right) N_{0}^{(1)} N_{0}^{(2)} \cosh k \cosh \kappa},
$$


where

$$
P=\prod_{n=1}^{\infty} \frac{\left(1+\mathrm{i} \kappa / k_{n}\right)\left(1+\mathrm{i} k / \kappa_{n}\right)}{\left(1+\mathrm{i} \kappa / \kappa_{n}\right)\left(1+\mathrm{i} k / k_{n}\right)}
$$

In order to obtain $R$ we return to the matching conditions on $x=\xi$. These can be combined to give

Consider now

$$
\sum_{n=0}^{\infty} \frac{V_{n}}{k_{0}+\kappa_{n}}=-\frac{2 \mathrm{i} k A_{0}}{\left(K_{2}-K_{1}\right) N_{0}^{(1)} \cosh k}
$$

$$
J=\frac{1}{2 \pi \mathrm{i}} \int_{C_{N}} \frac{f(z)}{z+k_{m}} \mathrm{~d} z
$$

with $f(z)$ as before. Cauchy's residue theorem now gives, for $m=0$,

$$
\sum_{n=0}^{\infty} \frac{V_{n}}{k_{0}+\kappa_{n}}=-A f\left(-k_{0}\right)
$$

Comparison with (B 19) then shows that

$$
R=\frac{k-\kappa}{k+\kappa} \mathrm{e}^{2 \mathrm{i} k \xi} \prod_{n=1}^{\infty} \frac{\left(1-\mathrm{i} k / k_{n}\right)\left(1+\mathrm{i} k / \kappa_{n}\right)}{\left(1-\mathrm{i} k / \kappa_{n}\right)\left(1+\mathrm{i} k / k_{n}\right)}=\frac{k-\kappa}{k+\kappa} \mathrm{e}^{2 \mathrm{ik} \xi} \mathrm{e}^{2 \mathrm{i} \theta}
$$

where

$$
\Theta=\sum_{n=1}^{\infty}\left\{\tan ^{-1}\left(\frac{k}{\kappa_{n}}\right)-\tan ^{-1}\left(\frac{k}{k_{n}}\right)\right\} .
$$

We can now express $T$ in a much neater form than (B 17). Using (2.8) and (B 22) it is clear that $|T|=2 k /(k+\kappa)$, and the phase of $T$ is just the phase of $P \exp \{\mathrm{i}(k-\kappa) \xi\}$. It is not difficult to show that

$$
\bar{P} / P=\mathrm{e}^{-2 \mathrm{i}(\theta+\theta)},
$$

where

$$
\theta=\sum_{n=1}^{\infty}\left\{\tan ^{-1}\left(\frac{\kappa}{k_{n}}\right)-\tan ^{-1}\left(\frac{\kappa}{\kappa_{n}}\right)\right\}
$$

Thus

$$
T=\frac{2 k}{k+\kappa} \mathrm{e}^{2 \mathrm{i}(k-\kappa) \xi} \mathrm{e}^{\mathrm{i}(\theta+\theta)}
$$

Equations (4.22)-(4.24) then follow.

\section{REFERENCES}

Chamberlain, P. G. 1993 Wave scattering over uneven depth using the mild-slope equation. Wave Motion 17, 267-285.

Devillard, P., Dunlop, F. \& Souillard, B. 1988 Localization of gravity waves on a channel with random bottom. J. Fluid Mech. 186, 521-538.

Evans, D. V. 1972 The application of a new source potential to the problem of the transmission of water waves over a shelf of arbitrary profile. Proc. Camb. Phil. Soc. 71, 391-410.

Evans, D. V. 1985 The solution of a class of boundary-value problems with smoothly varying boundary conditions. Q. J. Mech. Appl. Maths 38 (4), 521-536.

EvaNs, D. V. 1990 The wide-spacing approximation applied to multiple scattering and sloshing problems. J. Fluid Mech. 210, 647-658. 
Fitz-Gerald, G. F. 1976 The reflexion of plane gravity waves travelling in water of variable depth. Phil. Trans. R. Soc. Lond. 34, 49-89.

HAMILTON, J. 1977 Differential equations for long-period gravity waves on fluid of rapidly varying depth. J. Fluid Mech. 83, 289-310.

HeNRICI, P. 1974 Applied and Computational Complex Analysis, Vol 1. John Wiley and Sons.

JoHNSON, E. R. 1990 The low-frequency scattering of Kelvin waves by stepped topography. J. Fluid Mech. 215, 23-44.

KelleR, J. B. \& WeITz, M. 1953 Reflection and transmission coefficients for waves entering or leaving an ice field. Commun. Pure Appl. Maths 6, 415-417.

KIM, S. K., LIU, P. L.-F. \& LigGeTt, J. A. 1983 Boundary integral equation solutions for solitary wave generation. Coastal Engng 7, 299-317.

LAMB, H. 1932 Hydrodynamics, 6th edn. Cambridge University Press.

LeVINE, H. 1978 Unidirectional Wave Motions. North-Holland.

LINTON, C. M. \& Evans, D. V. 1992 Integral equations for a class of problems concerning obstacles in waveguides. $J$. Fluid Mech. 245, 349-365.

LiU, P. L.-F. \& LigGeTT, J. A. 1982 Applications of boundary element methods in problems of water waves. In Developments in Boundary Element Methods 2 nd edn (ed. P. K. Banerjea \& R. P. Shaw), Chap. 3, Applied Science Publishers.

Martin, P. A. 1984 Multiple scattering of surface water waves and the null-field method. Proc. 15th Symp. Naval Hydrodynamics, Hamburg, pp. 119-132. National Academy Press.

Mei, C. C. \& BlACK, J. L. 1969 Scattering of surface waves by rectangular obstacles in waters of finite depth. J. Fluid Mech. 38, 499-511.

MiLeS, J. W. 1967 Surface-wave scattering matrix for a shelf. J. Fluid Mech. 28, 755-767.

MitTra, R. \& LeE, S. W. 1971 Analytical Techniques in the Theory of Guided Waves. Macmillan.

Nachbin, A. \& Papanicolaou, G. C. $1992 a$ Boundary element method for long-time water wave propagation over rapidly varying bottom topography. Intl J. Numer. Meth. Fluids 14, 13471365.

Nachbin, A. \& Papanicolaou, G. C. $1992 b$ Water waves in shallow channels of rapidly varying depth. J. Fluid Mech. 241, 311-332.

NAMBA, M. \& FuKUSHIGE, K. 1980 Application of the equivalent surface source method to the acoustics of duct systems with non-uniform wall impedance. J. Sound Vib. 73, 125-146.

Newman, J. N. 1965 Propagation of water waves over an infinite step. J. Fluid Mech. 23, 399-415.

O'HARE, T. J. \& DAVIES, A. G. 1992 A new model for surface wave propagation over undulating topography. Coastal Engng 18, 251-266.

Roseau, M. 1976 Asymptotic Wave Theory. North-Holland.

URSELL, F. 1947 The effect of a fixed vertical barrier on surface waves in deep water. Proc. Camb. Phil. Soc. 43, 364-382.

Wehausen, J. N. \& Laitone, E. V. 1960 Surface Waves. Handbuch der Physik. Springer.

WeItz, M. \& KrLLER, J. B. 1950 Reflection of water waves from floating ice in water of finite depth. Commun. Pure Appl. Maths 3, 305-318.

YeUnG, R. W. 1975 A hybrid integral equation method for time-harmonic free surface flow. In Proc. 1st Intl Conf. on Num. Ship Hydrodynamics, pp. 581-607. 